\title{
Flow structures in a shallow channel with lateral bed-roughness variation
}

\author{
Yulia Akutina ${ }^{1, *}$, Frédéric Moulin ${ }^{2}$, Maxime Rouzes $^{2}$, and Olivier Eiff ${ }^{1}$ \\ ${ }^{1}$ Institute for Hydromechanics, Karlsruhe Institute of Technology, Karlsruhe, Germany \\ ${ }^{2}$ Institut de Mécanique des Fluides de Toulouse (IMFT), Université de Toulouse, CNRS, Toulouse, \\ France
}

\begin{abstract}
Highly heterogeneous floodplains can give rise to secondary flow structures responsible for the bulk of lateral momentum exchange. Quantifying the redistribution of momentum is required to predict lateral profiles of flow velocity and the associated water level in a river. In the work herein, we focus on studying secondary flow structures and the momentum redistribution associated with a lateral bed-roughness variation in a channel with low relative submergence of the roughness elements, $h / k=3,2$ and 1.5, where $h$ is the flow depth and $k$ is the roughness height. A series of laboratory experiments were performed in a flume containing rows of cubes. They were arranged in two types of regular patterns, with higher and lower frontal density, and placed side by side such that the bed roughness varies in the lateral direction. The measurements were performed using stereoscopic PIV in a vertical cross plane spanning between the two roughness types. The time-averaged and turbulence statistics of the three components of the velocity field were analyzed. First, we focus on the intensity of the secondary currents. As the flow becomes shallower (lower relative submergence), the cross-stream velocity normalized by the streamwise velocity increases. A large-scale secondary current at the border between the two roughnesses as observed in [1] (though in their case between smooth and rough regions) appears for $h / k=3$. As $h / k$ decreases, this structure reaches to the same size as the secondary flow generated by the roughness elements. Also, the discharge distribution between the two sides of the channel becomes less uniform with decreasing $h / k$. In this sense, the relative importance of the roughness difference increases with decreasing water depth. Moreover, higher discharge is observed on the side with higher equivalent sand roughness, contrary to what is observed for smooth-to-rough transition [1,2]. Time series of the streamwise velocity fluctuations are calculated using Taylor's "frozen turbulence" hypothesis. In this representation, streamwise velocity streaks are apparent for $h / k=3$, but they appear to lose coherence for the most shallow case of $h / k=1.5$.
\end{abstract}

\section{Introduction}

Predicting turbulence statistics, coherent structures, and secondary currents in shallow channel flows is important for quantifying momentum and mass transfer in rivers and flood plain

\footnotetext{
*e-mail: yulia.akutina@kit.edu
} 
flows. Application of three-dimensional numerical models is not always possible on large river reaches, but development of sound parametrization schemes can improve the accuracy of two-dimensional models that are commonly used. The FlowRes project (2015-2018) funded by the French National Research Agency (ANR) contributes to improving flood predictions and hazard assessment in floodplains. As part of this effort, in this work we focus on the flow structures associated with the lateral bed-roughness variation in flows with very low relative submergence of the roughness elements. This study case corresponds to an urbanized floodplain during extreme floods; hence the barely submerged roughness elements representing buildings, and the lateral roughness difference, corresponding, for instance, to different neighbourhoods.

The lateral momentum transfer due a roughness transition between smooth and rough bed in shallow open channels has been studied by [1,3]. Three mechanisms can drive the lateral mass and momentum exchange between the two parallel flows subjected to different bed roughness: (i) the secondary currents which develop due to transition, (ii) turbulence mixing due shear instability driven by the difference in speed of the flow over both sides, and (iii) a non-zero transverse mean when the flow is not uniform. Here we consider uniform flow.

It was shown by [1] that a secondary current develops over the smooth side of the channel and that it becomes more important in terms of its contribution to the lateral momentum exchange as the flow depth increases. In this study, however, both sides of the channel are fully rough and consist of large roughness elements. Also, the relative submergence is very low, $h / k=1.5-3$, where $h$ is the flow depth and $k$ is the roughness height, while in [1] the minimum submergence is about 11 .

Turbulent mixing can be drastically enhanced by the large-scale turbulent structures that result from a Kelvin-Helmholtz type shear instability. This phenomenon has been studied in plane shallow mixing layers by $[4,5]$ amongst others, concluding that the growth of the shear layer is suppressed by the bottom friction and therefore, the exchange due to KelvinHelmholtz turbulent structures is reduced in case of smaller depth, higher roughness and lower velocity difference between the parallel flows. On the other hand, [6] show that in a compound channel, where the velocity difference is sustained by the differences in roughness and flow depth, the large-scale Kelvin-Helmholtz structures persist even for very shallow conditions. They suggest that these structures are controlled solely by the mean flow distribution over the floodplain and the main channel. If the velocity ratio, $\lambda=\left(U_{1}-U_{2}\right) /\left(U_{1}+U_{2}\right)$ is larger than 0.3 and the mean velocity profile has an inflection point large-scale turbulent structures are observed.

Turbulent mixing is also realized through large- and very-large-scale motions that were measured in a laboratory rough-bed open-channel by [7] and in a river by [8]. These structures consist of streaks of higher and lower streamwise velocities and associated streamwise vortices. However, [9] suggests that the streaks tend to disappear for low relative submergence, when $h / k_{s}<2$, where $k_{s}$ is the equivalent sand-roughness.

It is thus clear that one can expect a large variety of flow-phenomenon to play a role in the current flow configuration. These secondary effects will result in the redistribution of the mean momentum in the channel cross-section affecting, among other things, the flowdepth. This work is devoted to measuring and analysing the distribution of the mean flow and secondary currents in the channel as a function of relative submergence.

\section{Methodology}

Stereoscopic PIV measurements were performed in a cross-plane of a $1.1 \mathrm{~m}$ wide and 26 $\mathrm{m}$ long flume with a slope of $0.3 \%$ to achieve uniform flow. The urban-type roughness 


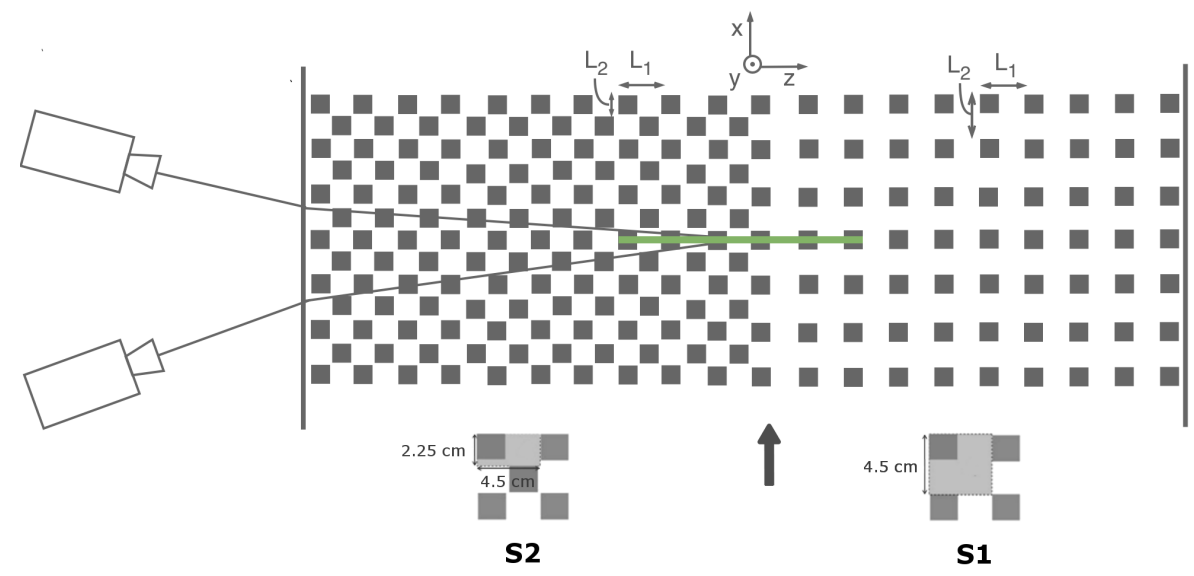

Figure 1. Schematic of the stereoscopic PIV set-up in the channel (top view). The measurements were taken in a vertical cross-flow plane (green line) spanning the two bed-roughness types with frontal densities $\lambda_{f}=0.4$ on the left- (S2), and $\lambda_{f}=0.2$ on the right-hand side (S1).

was modeled with cubes with a side of $h=0.02 \mathrm{~m}$ arranged in regular patterns. The lateral variation in the roughness was realized by the difference in roughness frontal density, $\lambda_{f}$, between the two half-sides of the flume. $\lambda_{f}=h^{2} / L_{1} L_{2}$ is equal to 0.4 on the left-hand side (referred to as S2 roughness type) and 0.2 on the right-hand side (referred to as S1) (Figure 1). Experiments were performed for three flow cases with varying relative submergence, $h / k=$ 3, 2 and 1.5, where $h$ is the flow depth and $k$ is the roughness height (Table 1). The three components of velocity were measured in a vertical cross-plane spanning the two roughness types (green line on Figure 1) with the frequency of $10 \mathrm{~Hz}$. The velocity vectors were calculated on a regular grid with the grid spacing of $0.66 \mathrm{~mm}$ in both vertical and horizontal direction using the software DaVis by LaVision. Further detail on the flow generation and stereoscopic set-up can be found in [10].

\section{Results}

An example of the instantaneous velocities obtained from the stereoscopic PIV measurements is given in Figure 2. The three plots correspond to the three different relative submergences. The streamwise velocity is shown as a colormap and the cross-plane velocities as vectors. It is clear that the flow is highly turbulent. It exhibits flow structures on the scale of the flow depth (e.g. a large counterclockwise structure at $y=0$ for $h / k=2$ ) and at the scale of the roughness

Table 1. Flow conditions for the three sets of experiments with varying relative submergence, $h / k$, where $h$ is the flow depth, $k$ is the roughness height, $F r$ is the Froude number, $Q$ is the total discharge, and $U_{\text {bulk }}$ is the bulk velocity calculated as $Q / A$, where $A$ is the cross-sectional area.

\begin{tabular}{lcccc}
\hline$h / k$ & $h(\mathrm{~cm})$ & $F r$ & $Q(\mathrm{~L} / \mathrm{s})$ & $U_{\text {bulk }}(\mathrm{cm} / \mathrm{s})$ \\
\hline 3 & 6.1 & 0.59 & 15.9 & 37.5 \\
2 & 4.1 & 0.53 & 5.3 & 23.9 \\
1.5 & 3.1 & 0.49 & 2.2 & 16.2 \\
\hline
\end{tabular}



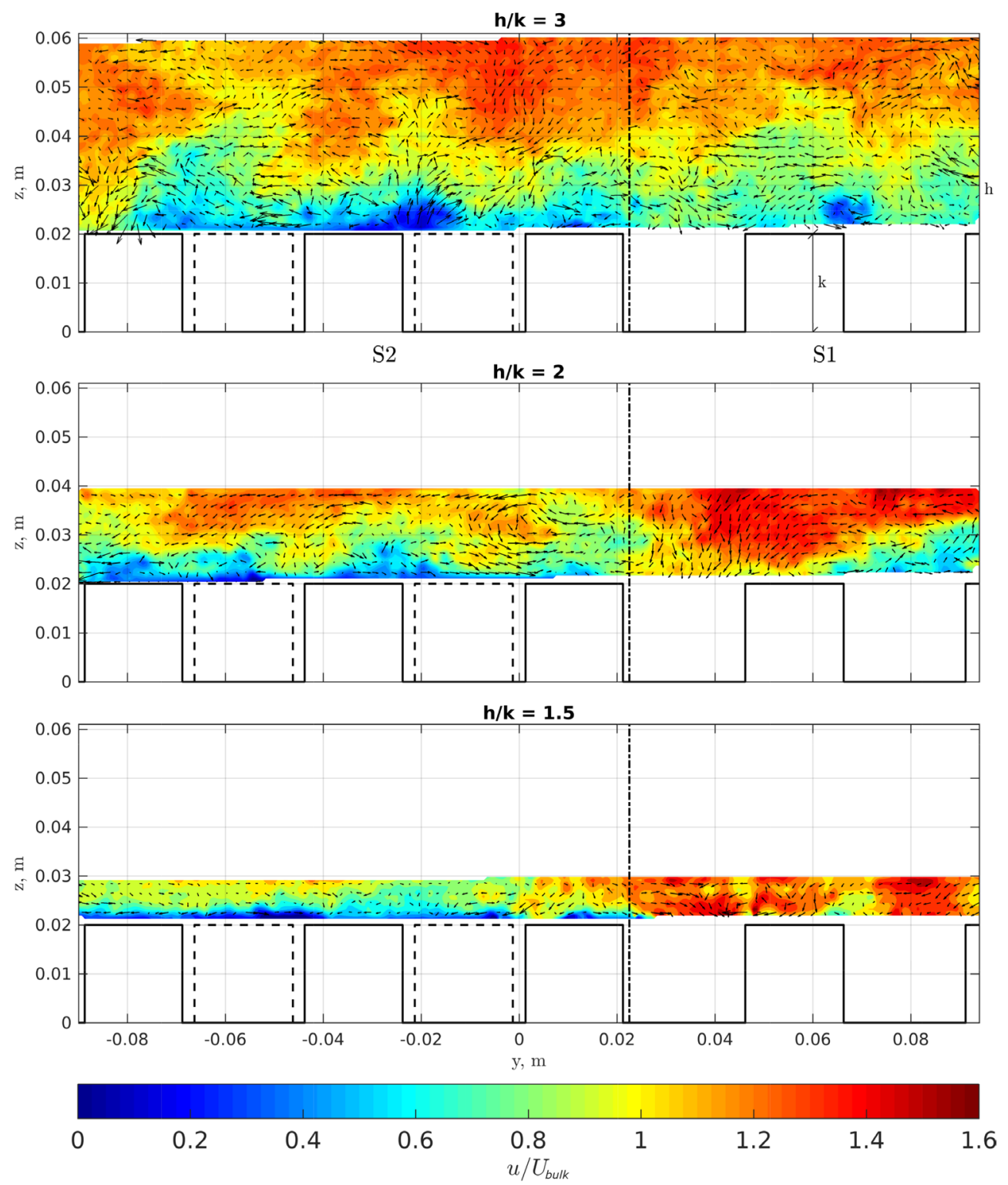

Figure 2. Instantaneous streamwise (colour) and cross-plane (vectors) velocities at the vertical crosssection for the three flow conditions with varying relative submergence, $h / k=3,2$ and 1 .

elements. It appears, that for deeper flow $(h / k=3)$ the effect of the roughness elements does not protrude all the way to the surface, while for $h / k=1.5$ the position of the cubes determines the distribution of the streamwise velocity up to the free-surface. Moreover, even from the instantaneous figures it is clear that the streamwise velocity is considerably higher over the S1 roughness (right-hand side) for lower submergences $h / k=2$ and 1.5, suggesting that lower relative submergence augments the importance of the roughness difference between the parallel flows. (This point will be discussed further in relation to Figure 4.) 
In order to separate the effects of the secondary currents and turbulence, the mean velocities $(U, V, W)$ were analysed first. Figure 3 shows the intensity of the secondary currents in the cross-section, measured as $I_{s c}=\sqrt{V^{2}+W^{2}} / U$, for different relative submergences; Figure 3a (depth-averaged) and Figure 3b (depth- and width-averaged over each roughness pattern). Figure 3 a reveals how the scale of the secondary currents changes as the flow becomes shallower. For deeper flow $(h / k=3$ and 2), the scale of the currents is approximately that of the flow depth. For the shallower case (blue line), they appear to be in phase with the roughness elements and their scale rather than corresponding to the flow depth. It is also striking how much more intense the cross-stream flow is in the lower submergence case. This point is illustrated even better in Figure $3 b$. The intensity of the secondary currents does not change significantly between the roughness types for $h / k=3$ and 2 . However, the case of $h / k=1.5$ appears drastically different from the other two with much higher overall secondary flow intensity and a significant difference between the roughness types. This is seemingly in contrast with higher submergence flows over smooth-to-rough lateral transitions. There, the large-scale secondary currents dominate and their influence decreases with decreasing $h / k$ ([1]). Yet, this can also be observed here: at $h / k=3$ the large-scale (depth-scale) secondary current's signature can be seen in Figure $3 \mathrm{a}$ between $\mathrm{y}=-0.02$ and $0.02 \mathrm{~m}$. As $h / k$ decreases,
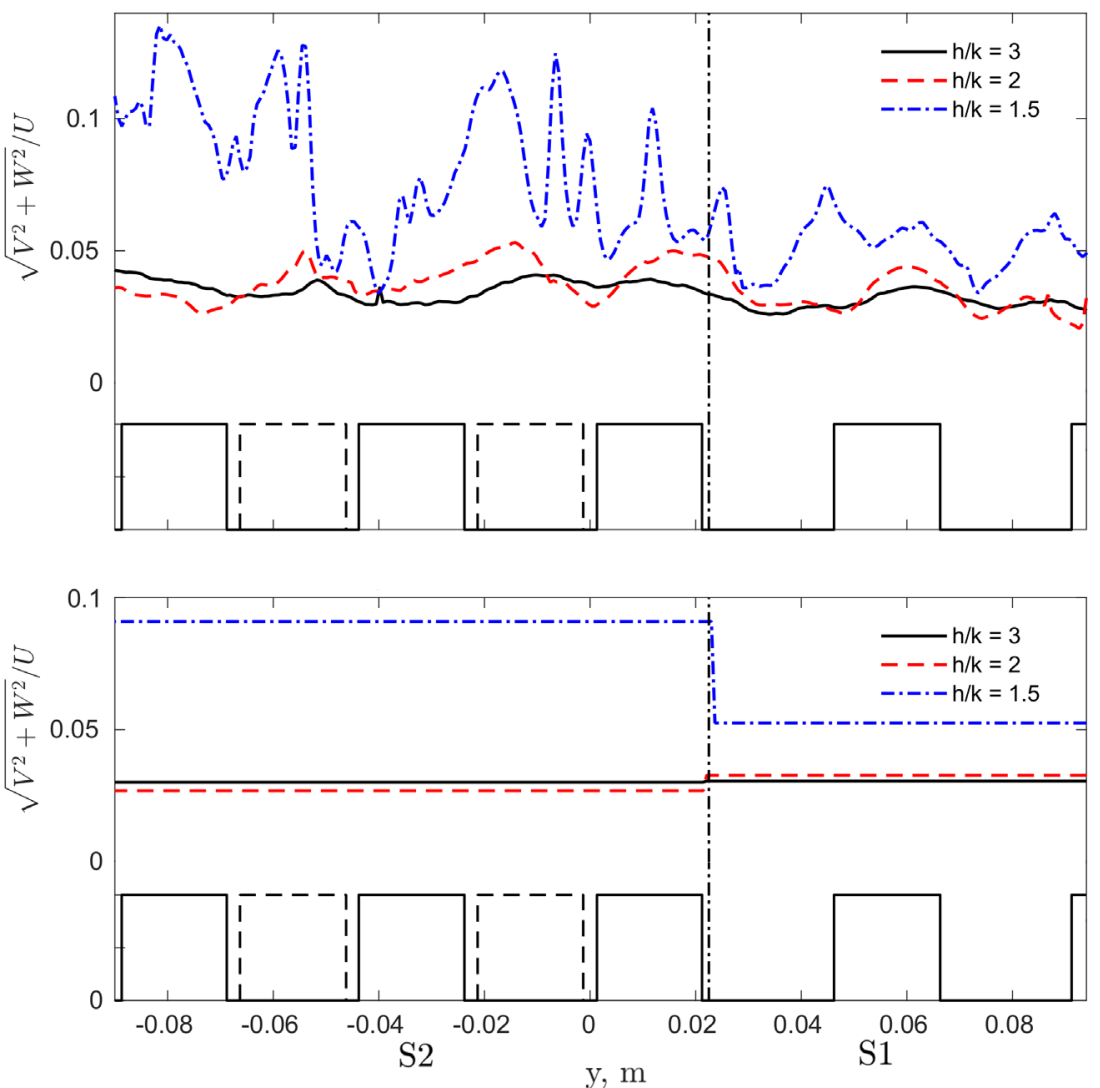

Figure 3. Relative intensity of the secondary currents, calculated as $I_{s c}=\sqrt{V^{2}+W^{2}} / U$, a) averaged over depth; b) averaged over depth and width for a given roughness type, for three relative submergences, $h / k=3,2$, and 1.5 . 
this structure becomes less pronounced since the flow is taken over by the roughness-scale secondary flows.

The loss of lateral homogeneity with decreasing relative submergence is also manifested in the mean flow distribution across the channel. Figure 4 shows the time-averaged streamwise velocity profiles across the roughness interface. For the deeper flow, the distribution is almost uniform with slight increase over S1. As the flow becomes shallower, the difference becomes markedly stronger; for $h / k=1.5$ the flow is considerably faster over S1. The $\lambda$-parameter, $\lambda=\left(U_{1}-U_{2}\right) /\left(U_{1}+U_{2}\right)$, which is a measure of asymmetry between parallel flows increases gradually as $h / k$ decreases: $\lambda=0.12,0.26$, and 0.42 for $h / k=3,2$, and 1.5 , respectively. According to [6], when $\lambda>0.3$, large-scale turbulent structures with a vertical axis of rotation develop due to Kelvin-Helmholtz type shear instability. A sufficient condition for it to appear is an inflection point in the streamwise velocity profile. However, in Figure 4, the blue line $(h / k=1.5)$ for which $\lambda>0.3$ does not resemble a classic shear-layer profile, but instead has numerous inflection points due to the roughness-scale secondary flows. It is thus not clear whether a large-scale horizontal turbulent structure appears in this case in spite of the large-scale gradient.

It is important to point out that contrary to what is expected from smooth-to-rough transitions, the higher velocities on the right-hand side of Figure 4 take place over the S1 roughness pattern which has a higher equivalent sand roughness (calculated through logarithmic law fits, see [10] for the method). Since considerably more flow is expected to pass through the alleys in S1 than through the thin slots in S2, it can be deduced that the total discharge is also higher for $\mathrm{S} 1$.

Streamwise velocity fluctuations in the horizontal $(x, z)$ planes were computed using Taylor's "frozen turbulence" hypothesis where the streamwise length $x$ is estimated from time $t$. Given that the advection velocity is much larger than the characteristic velocity of the turbulence, this is a reasonable hypothesis. In Figure 5, the streamwise velocity fluctuations are plotted for the three different $h / k$ for the same relative flow depth of $z / H_{e}=0.81$, where $H_{e}=H-k$ is taken as an effective flow depth. Flow faster then the mean is given in red, and slower in blue. In this representation, streamwise velocity streaks are apparent. In the

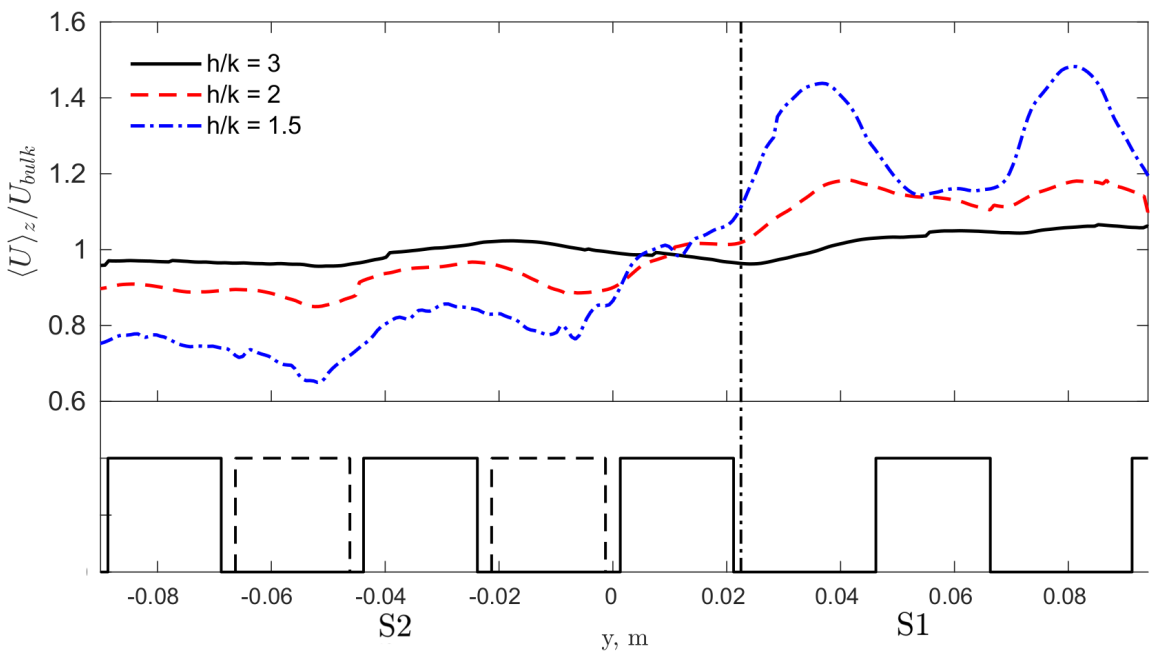

Figure 4. Time- and depth-averaged streamwise velocity profiles across the channel width at three different relative submergences, $h / k=3,2$, and 1.5 . 
deeper flow $(h / k=3)$, the structures appear elongated and are of the scale of about $10 H_{e}$ in length and $2 \mathrm{H}_{e}$ in width. As the flow becomes shallower, the structures break down and lose coherence. The "streaks" that now appear as blobs have the scale of about $4 H_{e}$ in both directions. One can also notice that the intensity of the streaks is lower on the S2 side (top of the plots) for lower relative submergence $(h / k=1.5$ and somewhat for $h / k=2)$, while for the deeper case the flow appears more homogeneous in the lateral direction. We also note that the streaks grow in width and length as they approach the water surface similar to the classic free boundary-layer case [e.g. 11] (results not shown).

\section{Conclusions}

Cross-plane stereoscopic PIV measurements were performed in an open channel with lateral roughness variation for very low relative submergences $(h / k=3,2$ and 1.5). The bedroughness on both sides consisted of cubes arranged on one side in a square configuration (S1) and on the other in staggered one (S2), with a frontal density $\left(\lambda_{f}\right)$ of $0.2(\mathrm{~S} 1)$ and 0.4 (S2). The lower frontal density (S1) has a higher equivalent sand roughness. The findings of this work can be summarized as follows.

It is observed that the lower the relative submergence, the more pronounced the effect of the roughness difference. This phenomenon is manifested in the distribution of the mean
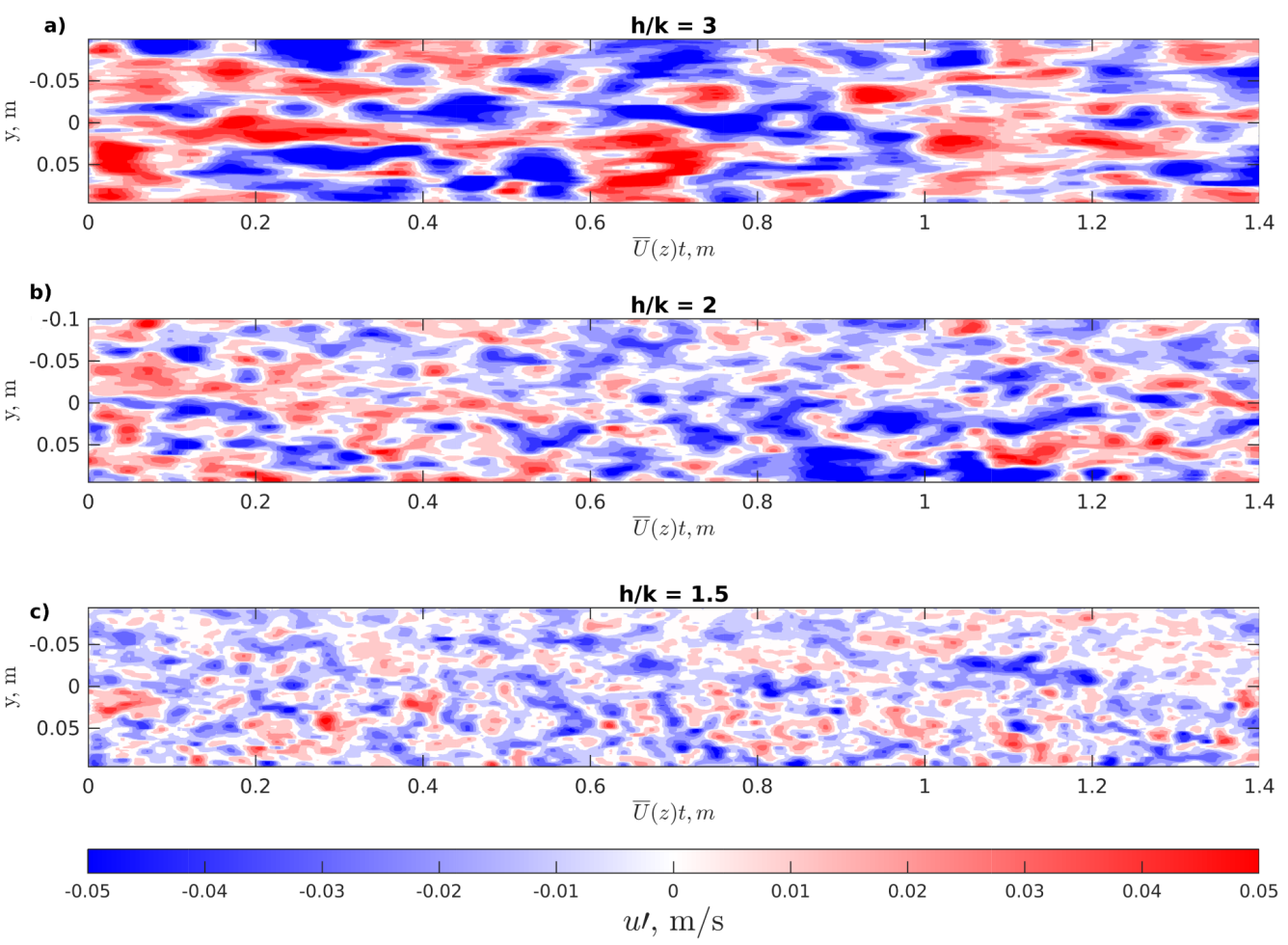

Figure 5. Streamwise velocity fluctuations, $u^{\prime}$, on a horizontal plane, $z=0.81 H_{e}$, plotted by converting the time coordinate $t$ into $x=\bar{U}(z) t$, where $\bar{U}(z)$ is the streamwise velocity averaged in time and width. Plots $\mathrm{a}, \mathrm{b}$ and c correspond to the three relative submergences, $h / k=3,2$, and 1.5 , respectively. 
flow across the channel, in the intensity of the secondary flows, and in the intensity of the streamwise velocity streaks, all of which lose their homogeneity in the lateral direction as the flow becomes shallower.

The instantaneous and secondary flow structures related to the roughness elements protrude all the way to the surface for $h / k=1.5$, while for $h / k=3$ the flow is relatively homogeneous in lateral direction closer to the surface.

Despite the widely accepted fact that in a shallower flow the lateral mixing is inhibited by the bottom friction (e.g., [4]), it is clear from the current results that the cross-stream variation intensifies as the flow becomes more shallow. This is due to the flow structures that develop on the scale of the roughness elements which become more prevalent than the large-scale flow structure for any low relative submergence.

At the three relative submergences considered here, all very low, a higher flow rate was observed on the side of the higher equivalent sand roughness (S1), contrary to what occurs for smooth to rough transitions, even for low relative submergence [2].

Streamwise velocity streaks are observed in Taylor's "frozen turbulence" representation of $x, y$ flow field. The streaks have a streamwise length of about $10 H_{e}$ in the deeper case, while they tend to break down and lose coherence when the flow becomes shallower. Also, the difference in streaks size and intensity on both sides of the transition also increases as $h / k$ decreases, i.e. both beds are increasingly locally controlled only. This suggests that a largescale mixing layer with large-scale turbulent structures is not present under very shallow conditions, in spite of higher large-scale shear with $\lambda>0.3$.

\section{References}

[1] D. Vermaas, W. Uijttewaal, A. Hoitink, Water Resour Res 47 (2011)

[2] V. Dupuis, F. Moulin, S. Cazin, M. Marchal, P. Elyakime, J.P. Barron, O. Eiff, Proceedings of the International Conference on Fluvial Hydraulics (River Flow 2018), LyonVilleurbanne, France (2018), (submitted)

[3] I. Kimura, W. Uijttewaal, Journal of Japan Society of Civil Engineering (JSCE) 2, 159 (2014)

[4] V.H. Chu, S. Babarutsi, J Hydraul Eng 114, 1257 (1988)

[5] W. Uijttewaal, R. Booij, Physics of Fluids 12, 392 (2000)

[6] S. Proust, J.N. Fernandes, J.B. Leal, N. Rivière, Y. Peltier, Water Resources Research 53, 3387 (2017)

[7] S. Cameron, V. Nikora, M. Stewart, J Fluid Mech 814, 416 (2017)

[8] M. Franca, U. Lemmin, Proceedings of the 4th IAHR Symposium on River, Coastal, and Estuarine Morphodynamics (RCEM 2005), Urbana, Illinois, USA 1, 203 (2005)

[9] A. Defina, Coherent flow structures in open channels (John Wiley \& Sons Ltd, London, U.K., 1996), pp. 87-99

[10] M. Rouzes, F. Moulin, E. Florens, O. Eiff, J Hydraul Res (2018), (in press)

[11] K. Kim, R. Adrian, Physics of Fluids 11, 417 (1999) 